\title{
¿DE QUÉ HABLAMOS CUANDO HABLAMOS DE BARRIO? TRAYECTORIA DEL CONCEPTO DE BARRIO Y APUNTES PARA SU PROBLEMATIZACIÓN
}

\author{
What we mean by neighbourhood? The trajectory of neighbourhood concept and \\ some notes for discussion
}

VERÓNICA TAPIA BARRÍA*

Fecha de recepción: 15 de marzo 2015 - Fecha de aprobación: 23 de mayo 2015

\section{Resumen}

La escala barrial se ha constituido como un elemento estratégico para las políticas urbanas al menos en los países de tradición occidental. Así, en Europa y Estados Unidos el barrio es la escala básica a partir de la cual se enfrentan objetivos tanto de regeneración urbana como también las agendas morales acerca de ciudadanía y cohesión. En Latinoamérica tampoco estamos lejos de ello, pues al menos desde la década de los noventa han surgido diversas políticas centradas en la escala barrial, especialmente en relación a los desafíos de revitalización de las áreas más empobrecidas de la ciudad. Sin embargo, la literatura denota una dificultad relevante relacionada con la escala de barrio: su ambigüedad conceptual. El objetivo de este artículo es entonces problematizar el concepto de barrio a partir de la contextualización de las políticas de escala barrial, la identificación de los principales elementos del que hemos denominado "concepto tradicional de barrio" y la presentación de apuntes conceptuales para un "concepto relacional de barrio".

Palabras clave: concepto de barrio, políticas urbanas, escala barrial.

\section{Abstract}

The neighbourhood has become a strategic element for urban policy at least in western states. Consequently, in Europe and the United States it is the foundation upon which urban regeneration goals, as well as moral agendas on citizenship and cohesion, are developed. Latin America follows a similar approach, from the nineties several policies focused on the neighbourhood scale have appeared, especially in relation to the renovation of the most impoverished areas of the cities. Nevertheless, there are one important difficulty related to the neighbourhood scale: its conceptual ambiguity. How face these obstacles? This article aims to discuss the neighbourhood concept based on a contextualization of the neighbourhood policies, the identification of the main elements of a "traditional concept of neighbourhood" and the presentation of conceptual approaches in order to propose a "relational concept of neighbourhood".

Keywords: neighbourhood concept, urban policy, neighbourhood scale.

* Antropóloga, Universidad de Chile. Doctorante en Geografía, Planificación Territorial y Gestión Ambiental Universidad de Barcelona. Correo electrónico:veronicatapiabarria@gmail.com 


\section{El Barrio como protagonista de las políticas urbanas de regeneración}

Si observamos el actual panorama internacional respecto a políticas urbanas nos encontramos con una serie de programas e iniciativas gubernamentales cuyo foco es el barrio, por ejemplo el New Deal for Communities (UK), Gran Projet de Ville, la Llei de Barris (Cataluña) y en Latinoamérica, el programa Quiero Mi Barrio (Chile) o el programa Mi Barrio (Perú). Todas estas políticas tienen un foco en común: están diseñadas con el objetivo de reducir la pobreza y desigualdad de las ciudades a partir de la intervención en barrios señalados como pobres o vulnerables. Si observamos la vasta experiencia de Estados Unidos en el diseño de políticas de foco barrial, podemos decir que esta tendencia centrada en el barrio es general al menos en los países de tradición occidental, desde los años noventa.

De este modo, tal como nos indica Atkinson et al. (2009), estas políticas sitúan a la escala barrial como el lugar a partir del cual distribuir servicios, intervenir en función de objetivos de regeneración y, lo más relevante, el barrio es aquella escala privilegiada donde los gobiernos definen sus agendas en relación a la definición y fortalecimiento de la cohesión social, capital social y ciudadanía.

La pregunta es entonces ¿por qué este protagonismo de la escala barrial? Nuestro planteamiento es que si bien ha habido una larga tradición de intervención a escala barrial, hay una conexión entre el reposicionamiento de la escala barrial y los procesos de reestructuración del capitalismo a escala global ocurridos justamente durante los ochenta y noventa. Esta vinculación tiene que ver con el hecho que el capitalismo de tipo fordista keynesiano o Estado de bienestar que primaría desde la posguerra sufriría justamente una crisis hacia fines de la década de los sesenta, produciéndose un proceso de reestructuración del capitalismo global en las décadas siguientes, que se ha calificado y conceptualizado entre otras categorías como posfordista, poskeynesiano o capitalismo de acumulación flexible (Soja, 2008:231-274).

Esta reestructuración la señalaremos aquí como neoliberalismo, definido como el régimen caracterizado por un marco institucional que privilegia derechos de propiedad privada, mercados libres fuertes, libertad de comercio y la relegación del rol del Estado a crear y reservar el marco institucional apropiado para el desarrollo de estas prácticas (Harvey, 2007: 8). Es justamente en este marco donde las políticas de escala barrial de la década de los noventa tendrán sentido: ¿de qué manera?

Sucede que la reestructuración neoliberal se puede entender en términos de etapas donde los organismos internacionales como el Fondo Monetario Internacional y el Banco Mundial han conducido las principales políticas económicas globales. Así, las reformas de primera generación implementadas a finales de los años ochenta y principios de los noventa se centraron en la estabilización macroeconómica y la liberalización económica como respuesta al modelo de desarrollo económico centrado sobre el Estado y las políticas de industrialización por substitución de importaciones. Sin embargo, a mediados de los años noventa se observa un cambio (Bustelo, 2003), avistado, por ejemplo, en el cambio en la presidencia del Banco Mundial una vez que asume Wolfensohn en 1995, y la declaración de los Objetivos de Desarrollo del Milenio por parte de las Naciones Unidas el año 2000. Se observa entonces 
un consenso entre los principales organismos internacionales en tanto las reformas de primera generación no habían cumplido satisfactoriamente con los resultados esperados pues no se habían logrado las expectativas de crecimiento económico, se enfrentaron periodos de crisis como la asiática de 1997, la pobreza seguía siendo un enorme problema especialmente en regiones como América Latina y fundamentalmente se reevalúa la pertinencia del Estado: si con las reformas de primera generación el ajuste estructural tenía por objetivo la reducción del Estado, la segunda generación de reformas va a abogar por un retorno del Estado, pero en los términos neoliberales. ¿Y por qué esto se plantea como un objetivo prioritario?, básicamente porque cuando amplios sectores de la población quedan marginados, los efectos colaterales ponen en peligro la estabilidad del proceso, por eso tanto la democratización como la modernización neoliberal en la región van a plantear la integración social como tarea prioritaria (Lechner, 2003: 6).

Así, el neo-consenso de Washington o el denominado Consenso de Santiago articulado durante la Cumbre de las Américas de 1998 se va a centrar en la reforma del Estado, el desarrollo institucional y el fortalecimiento de la gobernabilidad. La premisa de la segunda generación de reformas, es que las instituciones son cruciales para la estabilidad y que el Estado debe ser reconstituido para poder desempeñar sus responsabilidades pues la debilidad institucional constituye un importante obstáculo para las transformaciones económicas (Santiso, 2001), de manera que paradojalmente es necesario fortalecer el Estado para poder modificarlo, así mismo, el Estado es necesario para la estabilidad económica de corte neoliberal.
Sin embargo, en términos estrictos esta segunda ola de reformas es una manera de enfrentar el fracaso de los programas de ajuste estructural, intentar mejorar la vida de los países en desarrollo y contener los movimientos de protesta de las poblaciones afectadas, de modo que aunque las medidas adoptadas por el Banco Mundial y los organismos internacionales asociados se vincularon con temas de pobreza, medio ambiente, buena gobernanza, etc., en estricto rigor se continuó con los programas de ajuste en países como Sri Lanka, Ecuador, Haití, Ghana, Malí, Níger, la República Democrática del Congo y en el Chad (Toussaint, 2007).

En consecuencia, la persistencia e incluso el aumento de la pobreza, la desigualdad y las consecuentes tensiones sociales es un hecho conocido y finalmente asumido por los gobiernos neoliberales hacia fines de los ochenta y principios de los noventa y la atención se vuelve a poner en estos problemas que a menudo tenían su mayor expresión en las áreas urbanas, de esta manera, ninguna ciudad parecía ser inmune a los efectos de las modificaciones que estaban ocurriendo (Atkinson, 2000: 10371038). Es en este marco, entonces, que los gobiernos requieren enfrentar estos problemas con políticas específicas y es así como Europa (Unión Europea y Estados miembros) y Estados Unidos, lideran el proceso de diseño de una nueva generación de políticas urbanas donde el barrio adquiere un protagonismo central.

A partir de la década de los noventa se observa entonces una preponderancia de la escala barrial en una serie de políticas urbanas, mayormente diseñadas con el fin de enfrentar la pobreza y desigualdad de las ciudades y que tienen como foco principal el barrio, especialmente aquellos barrios identificados como 
pobres o vulnerables. De este modo, el barrio se constituye como foco para la distribución de servicios, también como base de intervención para objetivos de la tríada regeneración-rehabilitación-revitalización y finalmente, pero muy importante, a partir del foco en el barrio los gobiernos levantan sus agendas acerca de qué es cohesión social, capital social y ciudadanía (Atkinson et al., 2009:2818)

Este foco en el barrio se contextualiza entonces en las denominadas políticas o iniciativas de base local/territorial, conocidas mayormente como ABls o Area Based Iniciatives por su sigla en inglés. Este tipo de políticas que, si bien surgen en el contexto europeo como norteamericano, son una base influyente para el diseño de políticas tanto en Latinoamérica como incluso a una escala global.

Las ABls surgen en Europa como maneras de intervenir en conflictos raciales, dinámicas de desigualdad, segmentación social y falta de cohesión social lo cual se traduce en la consecuente segmentación del paisaje urbano, así, estos problemas generales se particularizan en una escala específica: los barrios o quartiers en crisis (Atkinson, 2007; Andersson \& Musterd, 2005). Una vez particularizados y expuestos en la prensa, los gobiernos se ven obligados a enfrentar esta fragmentación a partir de la modificación o incluso la creación de políticas que aborden estos temas de manera particularizada, levantando argumentos relacionados con la participación de la comunidad, el fortalecimiento del capital social y, en consecuencia, la cohesión social.

En Estados Unidos hay una larga tradición del enfoque barrial, pero ante problemas similares la actual perspectiva teórica es la del neighbourhood effect. Esta perspectiva enfatiza que el hecho de vivir en barrios pobres
- problemáticos afectará las perspectivas u oportunidades de vida en comparación a barrios mejores. Esto se aplica especialmente a temas como la educación, el crimen, la salud y especialmente a la problemática de la estigmatización. De este modo el diseño de políticas se enfoca justamente a reducir las concentraciones de pobreza, asimismo evitar la mala imagen de ciertos barrios y la promoción de la mixtura social.

Pero ¿qué es lo que implica barrio en el marco de estas políticas? La literatura sugiere varias cosas:

Primero, el barrio se configura como el bloque básico a partir del cual mantener la cohesión social (supuestamente en crisis); segundo, ante la evidente saturación de pobreza y desigualdad en zonas de las grandes ciudades el barrio se denota como el lugar de la comunidad local (Forrest, 2009); tercero, en el actual contexto global los gobiernos y los diseñadores de políticas públicas no son capaces de controlar los efectos del capitalismo global por lo cual el barrio es la escala más accesible y posible de intervenir (Kearns \& Parkinson, 2001); cuarto y final, el barrio ofrece una atractiva (y barata) alternativa para responder a la exclusión social y la regeneración urbana a través del fortalecimiento del capital social (Meegan \& Mitchell, 2001) y el gobierno local (Kennett \& Forrest, 2006).

De este modo, vemos que estas políticas de enfoque barrial básicamente conectan el barrio con los argumentos en pos de la cohesión social y capital social, lo cual ciertamente no se trata de ninguna casualidad. La literatura coincide que el modelo que impera en el diseño de las actuales políticas de enfoque barrial corresponde al desarrollado por el sociólogo norteamericano Robert Putman (Forrest \& Kearns, 2001), el cual contiene ciertas premisas: por una parte, se 
entiende capital social como aquellos elementos de la organización social que facilitan la cooperación y la ayuda mutua, los cuales son fundamentales para que las inversiones en capital físico y humano tengan mayores y mejores beneficios para las personas. Seguidamente, este proceso es fundamentalmente de abajo hacia arriba (bottom-up), es decir, que comunidades unidas, con un fuerte capital social contribuirán a la conformación de una sociedad cohesionada y ciudadanos participativos y activos.

De este modo, y bajo la perspectiva de este modelo, las políticas de enfoque barrial se basan entonces en dos argumentos conducentes a acciones específicas. El primer argumento es que si se fomenta o fortalece la cohesión social al nivel de barrio ello tendrá consecuencias positivas al nivel general de la sociedad, es decir, es una vía para la resolución de una crisis de la cohesión social. Y segundo, que si las comunidades excluidas y pobres generan capital y cohesión social ello tendrá como consecuencia su futura salida de su condición de exclusión.

Ahora, nuevamente es necesario preguntarse ¿son estas orientaciones y comprensión del problema una cuestión de azar? La respuesta nuevamente es negativa, y de acuerdo a nuestra perspectiva es necesario dar un paso hacia la identificación de los proyectos políticos o ideologías que promueven determinadas políticas. En este sentido, Forrest \& Kearns (Ídem) indican que los principales aspectos de esta orientación en el barrio son tres:

»Promoción del autogobierno ante la deslegitimación política.

»Promoción del sentido de pertenencia y arraigo al territorio.

»Fortalecimiento de los vínculos sociales y organizaciones comunitarias como una herramienta para salir de la exclusión social.
Son estos los recursos y herramientas que fundamentalmente concuerdan con el proyecto de la social democracia, por ejemplo el New Deal for Communities implementado por los laboristas en el Reino Unido, el Quiero Mi Barrio bajo los gobiernos de la concertación, la Llei de Barris del Partido Socialista de Cataluña y Grand Projet de la Ville bajo el gobierno socialista de Mitterrand.

Sin embargo, han sido numerosas las críticas que se han levantado en contra de estos argumentos en tanto la cohesión social no es siempre positiva, barrios fuertemente cohesionados podrían entrar en conflicto con otros contribuyendo a una ciudad fragmentada y dividida. Asimismo, la relación entre la supuesta crisis de cohesión social y la crisis de confianza y legitimidad política no es una relación directa, es decir, un barrio fuertemente cohesionado no necesariamente tiene un mayor compromiso democrático o más confianza en las instituciones, también -y muy relevante- fortalecer el capital social de barrios excluidos no implica de ninguna manera superar la condición de pobreza (Forrest \& Kearns, 2001). Finalmente, el uso, significado y rol del barrio varía enormemente, en consecuencia, estas políticas al considerar el barrio como el lugar de la comunidad local, como el lugar del capital y cohesión social no consideran esta diversidad (Kennett \& Forrest, 2006).

Justamente, es este último punto el que muestra un obstáculo básico al cual se han enfrentado los gobiernos a la hora de implementar políticas de enfoque barrial. Se trata del problema de la ambigüedad conceptual. 


\section{2. ¿De qué hablamos cuando hablamos de barrio?: ¿ambigüedad conceptual o consenso solapado?}

\footnotetext{
"No existe una definición exacta a propósito de qué es o qué conforma un barrio"1 (National Strategy Report, Social Exclusion Unit, Inglaterra, 2001).

"Para los efectos de esta ley se entiende por área urbana de atención especial el barrio o área urbana geográficamente diferenciable mayormente destinada a residencias habituales"2 (Llei de barris en Nelo, 2009).

"El primer problema que se enfrentó para la selección de los 200 barrios fue contar con una definición del concepto barrio. Se abrió una discusión al respecto entre los técnicos y, luego de un período de tiempo, no pudo concordarse una única definición”.

(Programa Quiero Mi Barrio, Ministerio de Vivienda y Urbanismo, Chile, 2008).
}

Estas citas reflejan una cuestión clave para las políticas de enfoque barrial, a saber, el problema de la definición del concepto de barrio y ciertamente no parece ser casualidad que estas tres experiencias reconozcan la complejidad que implica dicha tarea. En este marco resulta por lo menos curioso que la noción de barrio, nodal para el diseño e implementación de estas políticas, aparezca como una categoría de alguna manera dada, asumida y por tanto encubierta en una especie de ambigüedad.

Pero este problema no es nuevo ni tampoco se circunscribe solamente a la esfera de las políticas. Hace tres décadas el diagnóstico de Keller (1979) era que tanto en las investigaciones académicas como en la planificación urbana, el concepto de barrio es diverso y muchas veces incompatible. El diagnóstico actual tampoco varía mucho, en palabras de Galster:

"(...) los científicos urbanos han tratado el barrio como los jueces han venido tratando a la pornografía: como un término difícil de definir, pero que todos saben lo que es 'eso' cuando lo ven. Sin embargo, incluso una somera revisión de las definiciones de barrio que se encuentran en la literatura revela cruciales diferencias en lo que es 'eso' implícito" (2001:2111).

Asimismo, artículos más recientes destacan este problema, reafirmando que el barrio es un concepto vago y consecuentemente la mayoría de las investigaciones a menudo no proveen de un término con una definición explícita (Guo \& Bhat, 2007).

De este modo, respecto al renovado énfasis en el barrio por parte de las políticas urbanas, pensamos que tras esta ambigüedad conceptual existe efectivamente una construcción de un concepto de barrio, una noción que se ha ido configurado como hegemónica. Por lo tanto, esta noción de barrio como dada y asumida es, por el contrario, construida, en consecuencia es posible rastrear cómo el barrio ha adquirido ciertos atributos coherentes con su comprensión como base de la cohesión y capital social, como el lugar de la comunidad local.

\section{3. "Concepto de barrio tradicional": Escuela de Chicago}

Los principales atributos que se le han asignado al barrio y que son básicos para su comprensión en los términos de la generalidad de estas políticas son dos: el barrio como refugio de la comunidad y el barrio como unidad autocontenida, ambos -como veremos- se constituyen como una especie de legado de la Escuela de Chicago. 


\subsection{El barrio como refugio de la comunidad}

El concepto de comunidad desarrollado por esta escuela tiene unos atributos básicos, se trata de un grupo de personas y sus instituciones, localizadas en un área determinada (Park \& Burgess, 1984:115). Además, la comunidad desarrolla un tipo de cultura específica o modo de vida particular, a esto Burgess (Ídem: 145) lo denomina comunidad cultural, definida como los sentimientos, formas de conducta, vínculos y ceremonias características de una localidad.

Entonces ¿cuáles son las características de esta comunidad como modo de vida o cultura específica? Anderson lo llega a detallar bastante bien en su libro Sociología de la Comunidad Urbana, indicando que la comunidad se puede comprender como:

\begin{abstract}
“(...) una unidad global en la que existen diversos tipos de organización social, también como una localización $y$, asimismo, un lugar en que la gente encuentra los medios para vivir. Es un lugar no sólo de actividad económica y de asociación humana, sino también un lugar en el que se centran los recuerdos, tanto individuales como de grupo. Es más, la comunidad tiene la cualidad de la duración, que representa una acumulación de experiencias de grupo que vienen del pasado y se extienden a través de tiempo, aunque los individuos vayan y vengan siempre" (1965: 46-47).
\end{abstract}

De este modo, una comunidad se caracteriza por ser una unidad conformada por una organización social en una localización específica, donde la gente encuentra los medios para vivir, pero en la que también se genera una identidad y un sentido de pertenencia. Tomando en cuenta estas características principales, el barrio se entiende entonces como una comunidad en el pleno sentido del concepto en tanto es "una pequeña zona ocupada por un número limitado de gente que vive en una proximidad cerrada y en contacto frecuente, un grupo primario cara a cara" (Ídem: 61). De hecho, Burgess (1984: 147) llega a homologar el concepto de comunidad al de barrio al afirmar que "el barrio o la comunidad es el resultado de tres tipos de influencias: las ecológicas, las culturales y las políticas". Y es justamente este espíritu comunitario del barrio el que estaría en proceso de retirada debido a la aceleración de la vida urbana moderna. Anderson (1965), por ejemplo, observa desde una perspectiva diacrónica que el barrio tendría mayor presencia en el ámbito rural más que en el urbano, es decir, debido a la modernidad y la intensificación de los procesos de urbanización dicha dinámica estaría en franco proceso de extinción.

Es este proceso de debilitación del sentido comunitario y por ende del barrio, el que también expone Wirth en su clásico artículo sobre el modo de vida urbano, el cual justamente se caracterizaría por:

“(...) la sustitución de contactos primarios por secundarios, el debilitamiento de los vínculos de parentesco y la decadencia de la significación social de la familia, la desaparición del vecindario y la socavación de las bases tradicionales de la solidaridad social" (Wirth, 2010:12) ${ }^{3}$.

Park también evoca esta imagen de barrio y comunidad versus la desestructuración de la vida moderna, afirmando que:

\footnotetext{
“(...) en la ciudad el barrio tiende a perder gran parte de la significancia que poseía en sociedades más simples o primitivas. La facilidad de los medios de comunicación y transporte que permiten a los individuos distribuir sus intereses y vida en muchas partes al mismo tiempo tiende a destruir la permanencia e intimidad del barrio" (1925:9).
}

De este modo, entiende que el crecimiento de las ciudades implica la sustitución de las relaciones directas, cara a cara, primarias, 
por relaciones indirectas y secundarias (Ídem: 23). En otras palabras, el sentido comunitario como el elemento básico de la noción de barrio y, en consecuencia, la comprensión del barrio como una unidad geográfica conformada por un grupo de habitantes localizados en un espacio específico y cuya organización social se basa en la cooperación y la asociación, en los contactos frecuentes, cara a cara y donde se comparte un sentido de pertenencia e identidad es el que estaría en proceso de declinación debido a los nuevos modos de vida urbanos, vástagos de la modernidad. Y es aquí donde los aportes de los sociólogos clásicos completan el cuadro.

En síntesis, el barrio se constituye como el refugio del sentido comunitario, el cual está condenado a debilitarse o sencillamente desaparecer por la intensidad de la vida moderna y la intensificación del proceso de urbanización: el barrio como la última trinchera de resistencia de las relaciones de proximidad y los valores ligados al arraigo, la identidad, la memoria y la pertenencia.

\subsection{El barrio como unidad autocontenida}

El segundo elemento heredado de la Escuela de Chicago es el barrio como una unidad autocontenida, ya que tal como lo afirma Park:

"(...) con el paso del tiempo, cada sector o cada barrio de la ciudad adquiere algo del carácter y de las cualidades de sus habitantes. Cada parte distinta de la ciudad se colorea inevitablemente con los sentimientos particulares de su población. En consecuencia aquello que al principio solo era una simple expresión geográfica se transforma en un barrio; es decir, en una localidad con su propia sensibilidad, sus tradiciones y su historia particular" (Ídem: 6).
De este modo, podemos observar que junto con el sentido comunitario, el barrio se caracteriza por ser una parte distinguible y diferenciada de la ciudad, lo cual se vincula directamente con el concepto de área natural de la Escuela de Chicago. Las áreas naturales son definidas como "un área geográfica caracterizada a un tiempo por la individualidad física y por las características culturales de los individuos que en ella viven" (Zorbaugh citado en Theodorson, 1974:86) y básicamente son producto del crecimiento de la ciudad. Como puntualiza Burgess: "La expansión de la ciudad comporta un proceso de distribución que reorienta, distribuye y re-instala individuos y grupos por residencia y ocupación" (Burgess citado en Theodorson 1974:759).

El resultado es la diferenciación de la ciudad en áreas: las áreas naturales. Estas áreas "tienden a acentuar determinados rasgos, a atraerse y desarrollar sus tipos de individuo, a hacerse, por tanto, cada vez más diferenciadas" (Ídem: 75-76). En otras palabras, son unidades con características físicas, económicas y culturales distintivas y particulares. Consecuentemente, el barrio sería un área natural ya que es una unidad diferenciada de la ciudad, en cierto nivel independiente y autosuficiente: un receptáculo de una dinámica social y cultural específica.

En los años posteriores este modelo dual fue rechazado y los planteamientos de la Escuela de Chicago fueron fuertemente criticados, en el sentido de que la ciudad no es una expresión natural sino que es el resultado concreto de una forma de urbanización impulsada mediante la estructuración de procesos políticos, económicos y sociales (Castells, 1988:25). Críticas que no son ajenas a la escala barrial. 


\section{Críticas a la noción clásica de barrio: Ideología barrial}

Lefebvre constituye un referente en gran parte de las reformulaciones y planteamientos críticos de finales de la década de los sesenta, teniendo especial relevancia para una mirada crítica del concepto tradicional de barrio visto en el apartado anterior.

Sus argumentos en relación al barrio toman forma en su artículo publicado en el año 1967 en el cuaderno del L'Institut d'aménagement et d'urbanisme de la région parisienne, y posteriormente incluido en su versión al castellano en el libro $D e$ lo Rural a lo Urbano (1971). En este cuaderno dedicado al barrio y la ciudad, Lefebvre introduce la temática y los principales argumentos en relación a lo que él denomina la ideología barrial.

Esta ideología barrial responde a elementos del sentido común y por tanto no lograría separar los presupuestos de los hechos empíricos 0 cientíícos, es así como esta ideología identifica al barrio como la esencia de la realidad urbana y no como un aspecto contingente. La ideología barrial propone entonces organizar toda la vida urbana bajo el modelo del barrio, entendiéndolo como el ámbito natural de la vida y la unidad social a escala humana, "una especie de módulo social o sociológico, verificable y ratificable dentro de una exaltante unidad de juicios científicos y éticos, de conocimientos y de humanismo" (Ídem: 195).

El situar al barrio como la esencia de la ciudad expresa también otro error: la ingenuidad y el primitivismo antropológico y sociológico (Ídem: 197) que implica la recomposición o reconstrucción de la totalidad de la ciudad a partir de escalones o unidades cada vez más amplias (barrio, ciudad, región, nación), unidades que se definen a partir de la agrupación artificial de distintos elementos,
"(...) colecciones de cosas: viviendas, inmuebles, casas, calles y barrios, territorios y zonas de actividad que a continuación, por medio de una operación mágica, en nombre de vocablos como colectividad o comunidad se reintroduce en esta colección de cosas la conciencia, la vida. La operación es tan frecuente como grosera" (lbíd.)

Esta operación es la que se realiza menos con análisis de la especificidad social y más de acuerdo a un sentido nostálgico, romántico y valórico de arquitectos y urbanistas que -a juicio de Lefebvre- "toman esta ideología como concepción explicativa confundiendo su dogmatismo por una bien establecida verdad científica" (Ídem: 198) .

De este modo, la ideología barrial glorifica al barrio identificándolo como la base de la vida social, agrupa elementos sin mayor análisis y basado en nociones más bien valóricas para posteriormente asignarle una consciencia común: la vida de barrio. Vida de barrio que se erige como el modelo ético y valórico para organizar la ciudad. Ahora, ¿cuál es el aspecto primordial de este modelo?, no debería sorprender la conclusión del autor: "la ideología del barrio es una ideología comunitaria" (Ibíd.), es decir, el barrio como la fusión de actividades y conciencias que da lugar a una totalidad orgánica, en otras palabras, el barrio como una colectividad mediante la cual sus habitantes compartirían per se una conciencia común debido a las actividades que realizan en conjunto.

La crítica es aún mayor, pues esta colectividad iría subiendo de escalón de modo que de acuerdo a la ideología barrial, el sentido comunitario iría del barrio a la ciudad, de la ciudad a la región, de la región al país, etc., en un virtuoso espiral democrático, en palabras del autor: "esta ideología comunitaria se transforma en idealismo político, y un tipo ideal de vida social en utopía democrática" (Ibíd.). Utopía 
pues se creería que si se fortalecen los barrios se estaría fortaleciendo gradualmente este ideal comunitario-democrático. Nada más lejos de la realidad, pues, "en el barrio no se forman ni si instituyen los papeles sociales, las conductas o los comportamientos [...] el barrio no interviene en la proclamación de valores dominantes" (Ídem: 200).

En síntesis, la ideología barrial consiste en tres premisas a cuestionar:

1-. El barrio como una entidad que se auto explica por tanto aislada de los procesos sociales, económicos, políticos e incluso culturales más amplios.

2-. El barrio como un ideal de una vida comunitaria siempre armoniosa, sin conflictos.

3-. La capacidad del barrio de contagiar estos valores.

De esta manera, la noción clásica de barrio es cuestionada porque se constituye como un ideal, como la escala preferente donde el sentido comunitario, la solidaridad, la identidad e incluso donde los valores democráticos preferentemente encuentran su sitio; esto a pesar e incluso en contraposición a los procesos de disociación, caos y anonimato que según la noción clásica caracterizarían el conjunto de la ciudad.

Así, esta crítica permite por una parte rechazar el barrio como una realidad esencial, pero también problematizarlo en base a evaluar el grado de realidad de una serie de afirmaciones que Lefebvre resume de la siguiente manera:

a) El barrio es una unidad sociológica relativa, subordinada, que no define la realidad social, pero que es necesaria. Sin barrios, igual que sin calles, puede haber aglomeración, tejido urbano, megalópolis, pero no hay ciudad. El barrio coincide con un espacio geométrico, euclidiano, ordenado, homogéneo, pero es también donde el espacio y el tiempo social dejan de ser orgánicos y organizados, y abre el paso a modificaciones y cualificaciones de los habitantes.

b) El barrio tiene una existencia a medias, simultáneamente para el habitante y para el sociólogo. Es donde se constituyen relaciones interpersonales más o menos duraderas y profundas. La condición de espacio intermedio entre el espacio geométrico y el espacio social implica que la proximidad en el espacio y el tiempo (proximidad geométrica, por ejemplo el hecho de habitar el mismo espacio con otro sujeto de otra clase o generación), sustituye las distancias sociales, espaciales y temporales (espacio social). Es por ello que el barrio constituiría un umbral en la expresión y la existencia sociológica: el tránsito de lo accesible al individuo en tanto vinculado al suelo -espacio geométrico (el habitante)- a lo inaccesible en cuanto a individuo vinculado a un espacio y tiempo social.

c) El barrio como el microcosmos de un peatón que recorre un cierto espacio en un tiempo determinado sin tener necesidad de usar vehículo motorizado. Es en relación a este hecho cotidiano de un ciudadano a pie que se delinea un área o radio de acción, en base al cual se ha producido historia y además se produce un cierto reparto de actividades, sobre todo en zonas comerciales, de intercambio, de relación y de comunicación. Aquí es también donde se expresa esta naturaleza liminal del barrio, en tanto estas actividades están determinadas por una parte por la sociedad en su conjunto, pero también por las exigencias de la vida inmediata y cotidiana. Es en este sentido que le corresponde al barrio un equipo más o menos suficiente y 
completo, que no lo hace autosuficiente, pues estos equipamientos dependen de grupos funcionales más amplios, activos a escala de la ciudad, de la región, del país etc., dependiendo completamente de otras estructuras más vastas como municipalidades, poder político, instituciones.

d) En consecuencia, el barrio es una ínfima malla del tejido urbano y de la red que constituye los espacios sociales de la ciudad. Esta malla por tanto puede romperse, sin que el tejido sufra daños irreparables, pues otras instancias pueden entrar en acción y suplir sus funciones.

e) Sin embargo, el barrio es también el nivel donde el espacio y el tiempo de los habitantes toman forma y sentido en el espacio urbano.

Es en base a la pertinencia de estas afirmaciones que se puede abrir la discusión y reflexión en relación al barrio, superando la ideología barrial en tanto el autor propone realizar análisis concretos, en una teoría del conjunto, con conceptos del espacio y el tiempo sociales. Así, propone una serie de investigaciones concretas, que se sintetizan principalmente en: definir un estándar de dotaciones que permitan consolidar las unidades barriales; desarrollar una tipología de los barrios en base al inventario y comparación del equipamiento clasificando los barrios en diferentes tipos (por ejemplo los que se mantienen, los consolidados, los que desaparecen) y, finalmente, estudiar las imbricaciones y relaciones internas y externas entre los barrios y lo que les rodea.

En síntesis, el estudio debería ser capaz de proponer los criterios de existencia y de cohesión de este núcleo parcial urbano a partir de un estudio tipológico complementado con un estudio de tendencia general de los barrios (¿los barrios se consolidan o desaparecen?, ¿Cómo se relacionan los barrios con las tendencias de la ciudad como conjunto?) lo que a su vez implica el estudio del territorio que la rodea y la planificación de este (Ídem: 202 y 203).

\section{Una mirada alternativa: concepto de barrio relacional}

Retomando entonces la crítica de Lefebvre, podemos resumir los principales rasgos de la ideología barrial en cuatro ideas:

Primero, el barrio y su condición intrínseca de lugar, por lo cual se constituye como una unidad distinguible y delimitada en el conjunto de la ciudad, contenedor de una identidad única y particular.

Segundo, la superposición directa de las categorías lugar-comunidad-identidad. De este modo, el barrio al constituirse como un lugar, forzosamente está asociado a una comunidad específica y por ende a una identidad particular compartida, única.

Tercero, el barrio es una escala local y por tanto es un lugar, en contraposición a la dimensión global.

Cuarto, el barrio -en su calidad local y condición de lugar en oposición al espacio globalse constituye como el refugio, la trinchera de defensa de la identidad y de la comunidad frente a unas fuerzas globales abstractas, externas, poderosas y potencialmente desintegradoras. $Y$ esto es válido tanto en relación a la modernización-urbanización como la actual globalización.

De esta forma, interesa especialmente proponer algunos elementos claves en pos de problematizar el concepto de barrio lo cual exige poner en cuestión básicamente estos cuatro puntos, y es aquí donde los argumentos de D. Massey (1994 y 2004) son especialmente esclarecedores. Respecto al primer punto señalado en el apartado anterior, el barrio como lugar no se 
caracteriza por el hecho de tener una identidad propia, inmóvil, fija y característica, ni tampoco es aquello que está dentro de unos bordes o delimitaciones. Por el contrario, el barrio como lugar se puede comprender como un punto de intersección de relaciones sociales en un momento dado, relaciones sociales que se extienden a una escala mayor que la que definen ese lugar en ese preciso momento. Esto implica abrir el barrio tanto en el tiempo como en el espacio, es decir que el barrio se construye y se modifica en relación al presente, al pasado y también al futuro, pero también en cuanto a la proyección de esta intersección de relaciones sociales a todas las escalas. Asimismo, esta perspectiva enriquece el concepto pues considera los conflictos y "la necesidad de negociar a través y con la diferencia el implacable hecho espacial de compartir un terreno" (Massey, 2004:6).

En cuanto al segundo punto, cabe preguntarse ¿Es que el lugar forzosamente está definido por una comunidad que a la vez comparte una identidad única y particular? Esto es particularmente relevante pues si observamos con atención "raramente comunidad y lugar son co-términos" (Massey, 1994:147), más aún, "las comunidades pueden existir sin compartir el mismo lugar" (Ídem: 154), asimismo, es improbable que una comunidad sea un grupo social coherente, homogéneo con el mismo sentido de lugar. De este modo, un barrio puede estar constituido por distintas identidades, por ejemplo en relación al género, la edad o la actividad política.

Relacionado con el tercer punto, es necesario dar cuenta que existe una narrativa dominante que ha reforzado la idea de una contraposición entre el lugar (asociándose a lo local) y el espacio (asociándose a lo global), donde el lugar-local es más significativo que el espacio-global. En este sentido, el barrio sería aquello real, territorialmente emplazado, cotidiano y vivido, en contraposición a un espacio global que está en algún lado, afuera, omnipresente y abstracto. Pero el espacio global es tan real y cotidiano como el lugar, es la suma de relaciones, conexiones, personificaciones y prácticas, pero que son completamente cotidianas y emplazadas al mismo tiempo que en conjunto van alrededor del mundo (Massey, 2004:8).

Finalmente y en cuanto al último punto, cuestionar este binomio global/local - espacio/lugar implica que el barrio no se defiende de unas fuerzas globales que están ahí fuera, muy por el contrario, los lugares son momentos donde lo global se constituye, inventa, coordina y produce, son agentes en la globalización (Ídem: 11). Esto significa que el barrio como lugar tiene posibilidades de acción que van mucho más allá de defenderse de lo global, por el contrario, tiene responsabilidad en el actual estado de cosas y, por lo mismo, tiene posibilidad de modificarlo.

\section{Propuesta}

El desafío es entonces conceptualizar el barrio en términos relacionales y poder plantear ciertos criterios de definición que no impliquen un encapsulamiento, una frontera entre un ellos y un nosotros, entre un dentro y un fuera,

La propuesta es entonces distinguir entre una definición conceptual (el barrio abierto y relacional) y una definición operativa. La definición operativa implica la delimitación del barrio asumiendo justamente que se trata de una construcción derivada de decisiones que en último término están insertas en geometrías de poder (Massey citada en Bird et al., 1993). 
Nuestra propuesta de definición de barrio entonces parte de conceptualizarlo como un lugar, una espacialidad urbana, en el sentido de ser una particular constelación de relaciones sociales que se encuentran y vinculan en un locus particular, en este caso, el barrio. Asimismo, el barrio lejos de tener como particularidad el contener una única identidad explicada en base a su historia, diremos que más bien su particularidad está dada por ser el punto de encuentro de la heterogeneidad, pero ser un único encuentro de múltiples trayectorias en un momento dado, un encuentro de múltiples identidades forjadas en relación y que se encuentran en el barrio, pero se proyectan más allá.

Ahora ¿Qué es lo que diferencia al barrio de otras identidades espaciales? El barrio, entendido como lugar, es donde de manera predominante se desarrollan aspectos de la vida cotidiana de esas identidades, de esos sujetos que ahí se encuentran, es decir, que este encuentro de trayectorias en el caso del barrio se daría principalmente por el hecho de compartir más o menos permanentemente aspectos relacionados directamente con la vida cotidiana y los espacios donde ésta se desarrolla, más aún, aspectos específicos de la vida cotidiana que tienen que ver con el hecho de compartir ciertos espacios con otros que viven o residen en condiciones de proximidad geográfica.

En síntesis: el barrio como el lugar de encuentro más o menos estable y más o menos permanente de distintas trayectorias caracterizado principalmente por el hecho de compartir algunos de los aspectos de la vida cotidiana y sus espacios asociados, específicamente aquellos aspectos que se derivan del hecho de vivir o residir en condiciones de proximidad geográfica. Pero, ¿cuál es esta proximidad geográfica? A la luz de Lefebvre podríamos enunciar que dicha proximidad geográfica podría estar definida por la escala peatonal, es decir, por un área que en promedio es posible de recorrer a pie. Asimismo, la vida cotidiana que se desarrolla en el barrio al menos da cuenta de la necesaria existencia de equipamientos mínimos para su desarrollo.

Además, cabe preguntarse ¿Cuál es la relación del barrio con los conceptos de comunidad y sentido de lugar? Es en este punto que nos confrontamos con las herencias y con las nuevas perspectivas trabajadas en estas páginas.

Teniendo en mente la perspectiva de Massey asumimos en primer lugar que el barrio como lugar, per se no se constituye como el contenedor de una identidad única y particular construida por una historia común; de una comunidad que comparte un único sentido de lugar, unos valores, unas tradiciones, un sentido de pertenencia absoluto, etc. Por el contrario, nuestros referentes de identidad son múltiples, diversos, extensos y en continua construcción; el sentido de pertenencia, de arraigo, de valores comunes no es el patrimonio del lugar, sino que también es global, la vida cotidiana, sus aprendizajes y atributos se extienden de lo global a lo local y de lo local a lo global.

De modo que nos planteamos estos aspectos como una posibilidad, una posibilidad que implica asimismo una responsabilidad, una actitud activa frente a los hechos que nos sucede aquí y ahora, aquí y ahora en el barrio, pero también a todas las escalas. En otras palabras, los atributos de identidad, sentido de pertenencia, valores y sentidos compartidos, arraigo, etc., agrupados en el concepto de comunidad y de sentido de lugar diremos que para el barrio son una posibilidad.

Esto implica asumir que el encuentro más o menos permanente, más o menos estable 
de trayectorias que conforman ese espacio, ese lugar denominado barrio, caracterizado por el hecho de compartir con otros que viven o residen en proximidad geográfica ciertos aspectos de la vida cotidiana y sus espacios asociados, implica también conflicto y con ello la posibilidad de negociación. La posibilidad de la construcción de un sentido colectivo en el barrio, la posibilidad de compartir ciertos aspectos de sentido de lugar, de valores, de historia, de tradiciones, de organización, de apuestas políticas de cómo y por qué pensamos serían mejor las cosas, y ello claramente puede encontrarse en el barrio, pero al mismo se proyecta a todos los ámbitos de nuestras existencias. Esta visión local es global, y lo global es interceptado por lo local.-

\section{Notas}

1 "There is no exact definition of what makes a neighbourhood".

2 "Als efectes d'aquesta Llei, s'entén per àrea urbana d'atenció especial el barri o l'àrea urbana geogràficament diferenciables, majoritàriament destinats a habitatges habituals".

${ }^{3}$ El destacado es nuestro.

\section{Referencias bibliográficas}

Anderson, N. (1965). Sociología de la Comunidad Urbana. Ciudad de México: Fondo de Cultura Económica.

Andersson, R. \& Musterd, S. (2005). "Area-Based Policies: A Critical Appraisal" Tijdschrift voor Economische en Sociale Geografie, ํo 96 , № 4.

Atkinson, R. (2000). "Combating Social Exclusion in Europe: The New Urban Policy Challenge”. Urban Studies, № 37.

Atkinson, R. (2007). EU Urban Policy, European Urban Policies and the Neighbourhood: An overview of concepts, programmes and strategies. Paper presented at a EURA Conference - The Vital City, Glasgow, September 12th-14th.

Atkinson, R., Dowling, R. \& McGuirk, P. (2009). "Home/ Neighbourhood/City/+". Environment and Planning, ํo41, № 12.

Bird, J., Curtis, B., Putman, T. \& Tickner, L. (2005). Mapping the Futures: Local Cultures, Global Change. London: Taylor \& Francis.

Bustelo, P. (2003). Desarrollo económico: del consenso al post consenso de Washington y más allá. En Estudios de historia y de pensamiento económico: homenaje al profesor Francisco Bustelo García del Real. Madrid: Editorial Complutense.

Castells, M. (1988). Problemas de Investigación en Sociología Urbana. Ciudad de México: Siglo XXI.
Dietz, R. (2002). "The estimation of neighborhood effects in the social sciences: An interdisciplinary approach". Social Science Research, № 31 .

Forrest, R. (2008). "Who Cares About Neighbourhoods?". International Social Science Journal, № 59, № 191.

Galster, G. (2001). "On The Nature of Neighbourhood". Urban Studies, ํo38, № 12.

Guo, J. \& Bhat, Ch. (2007). "Operationalizing the concept of neighborhood: Application to residential location choice analysis". Journal of Transport Geography, № 15.

Harvey, D. (2007). Breve historia del neoliberalismo. Madrid: Akal.

Kearns, A. \& Parkinson, M. (2001). "The Significance of Neighbourhood". Urban Studies, №38, № 12.

Keller, S. (1979). El Vecindario Urbano Una Perspectiva Sociológica. Ciudad de México: Siglo XXI.

Kennett, P. \& Forrest, R. (2006). "The Neighbourhood in a European Context”. Urban Studies, ํo43, № 4.

Lechner, N. (2003). "Estado y Sociedad en una perspectiva democrática". Polis № 6.

Lefebvre, H. (1975). De lo Rural a lo Urbano. Barcelona: Península. 
Massey, D. (2004). "Geographies of Responsibility". Geografiska Annaler, № 86.

Meggan, R. \& Mitchell, A. (2001). "It's Not Community Round Here, It's Neighbourhood': Neighbourhood Change and Cohesion in Urban Regeneration Policies". Urban Studies, № 38, № 12.

Ministerio de Vivienda y Urbanismo del Gobierno de Chile (2008). Programa Quiero Mi Barrio.

Nelo, O. (2009). La Llei de barris, una aposta collectiva per la cohesió social. Barcelona: Departament de Política Territorial i Obres Públiques.

Park, R. \& Burgess, E. (1984). The City. Suggestions for Investigation of Human Behavior in the Urban Enviroment. Chicago: The University of Chicago Press.
Santiso, C. (2001). "Gobernabilidad democrática y reformas económicas de segunda generación en América Latina". Instituciones y desarrollo, № 8 .

Social Exclusion Unit (2001). A New Commitment to Neighbourhood Renewal - National Strategy Action Plan. U.K: Social Exclusion Unit.

Soja, E. (2008). Postmetrópolis. Estudios críticos sobre las ciudades y las regiones. Madrid: Traficantes de Sueños.

Theodorson, G. (1974). Estudios de Ecología Humana. BarceIona: Labor.

Toussaint, E. (2007). Banco Mundial: el golpe de estado permanente: la agenda oculta del Consenso de Washington. España: Editorial EI Viejo Topo. 